UOSTP-00-106

hep-th/0008204

\title{
Exact Solutions of Multi-Vortices and False Vacuum Bubbles in Noncommutative Abelian-Higgs Theories
}

\author{
Dongsu Bakf \\ Physics Department, University of Seoul, Seoul 130-743 Korea
}

\begin{abstract}
We consider the noncommutative Abelian-Higgs theory and construct new type of exact multi-vortex solutions that solve the static equations of motion. They in general do not follow from the BPS equations; only for some specific values of parameters, they satisfy the BPS equations saturating the Bogomol'nyi bound. We further consider the Abelian-Higgs theory with more complicated scalar potential allowing unstable minima and construct exact solutions of noncommutative false vacuum bubble with integer magnetic flux. The classical stability of the solutions is discussed.
\end{abstract}

*Electronic Mail: dsbak@mach.uos.ac.kr 
The ordinary Abelian-Higgs theory possesses all the essential ingredients leading to the spontaneous symmetry breaking via the condensation of the scalar field, serving as a model for superconductivity. The Maxwell field as well as the Higgs field become massive and magnetic fluxes are confined. The theory allows the topological vortex solution carrying $m$ unit magnetic fluxes唒. Especially in the critical case, there exist static multi-vortex solutions saturating the BPS bound. However, until now, no closed form vortex solutions are found in this theory. Recently, some aspects of the Nielsen-Olesen vortices in the noncommutative Abelian-Higgs theory have been investigated via the BPS equations [2, 3] and related works have appeared [4, 5].

In this letter, we shall consider the noncommutative Abelian-Higgs model. (The gauge symmetry is in fact non-Abelian due to the noncommutative nature of the theory.) Our key finding is new types of exact multi-vortex solutions. When the mass parameter of the Higgs field takes a certain value, these solutions saturate the BPS bound and the classical stability is guaranteed in this case. We discuss the detailed characteristics of the vortex solutions mainly focused on the static properties. In particular, the gauge fields arising here give localized integer-valued fluxes, which are also static solutions of the noncommutative Maxwell theory [4].

We further consider the Abelian-Higgs theory with more complicated scalar potential allowing unstable minima and construct exact solutions of noncommutative false vacuum bubble[6] with integer magnetic flux. We close our discussion by mentioning related higher dimensional solutions and possible applications.

Vortices in Noncommutative Abelian-Higgs Theory: We shall begin with the noncommutative Abelian-Higgs model in 2+1 dimensions described by [2, 3]

$$
L=-\frac{1}{g^{2}} \int d^{2} x\left(\frac{1}{4} F_{\mu \nu} * F^{\mu \nu}+D_{\mu} \phi *\left(D^{\mu} \phi\right)^{\dagger}+\frac{\lambda}{2}\left(\phi * \phi^{\dagger}-v^{2}\right)^{2}\right)
$$

where

$$
\begin{aligned}
F_{\mu \nu} & =\partial_{\mu} A_{\nu}-\partial_{\nu} A_{\mu}-i\left(A_{\mu} * A_{\nu}-A_{\nu} * A_{\mu}\right) \\
D_{\mu} \phi & =\partial_{\mu} \phi-i A_{\mu} * \phi
\end{aligned}
$$

and our metric convention is $\eta_{\mu \nu}=\operatorname{diag}(-1,1,1)$. Here the $*$-product is defined by

$$
\left.f(x) * g(x) \equiv\left(e^{-i \frac{\theta}{2} \epsilon^{i j} \partial_{i} \partial_{j}^{\prime}} f(x) g\left(x^{\prime}\right)\right)\right|_{x=x^{\prime}},
$$

where we take $\theta$ to be positive without loss of generality. The theory can be equivalently presented by operators on the Hilbert space defined by

$$
[\hat{x}, \hat{y}]=-i \theta
$$

where the *-product between functions becomes the ordinary product between the operators. For given function

$$
f(x, y)=\int \frac{d^{2} k}{(2 \pi)^{2}} \tilde{f}(k) e^{i\left(k_{x} x+k_{y} y\right)},
$$

the corresponding operator can be found by the Weyl-ordered form of

$$
\hat{f}(\hat{x}, \hat{y})=\int \frac{d^{2} k}{(2 \pi)^{2}} \tilde{f}(k) e^{i\left(k_{x} \hat{x}+k_{y} \hat{y}\right)} .
$$


One may then easily show that $\int d^{2} x f$ is replaced by $2 \pi \theta \operatorname{tr} \hat{f}$ and $\partial_{i} f$ corresponds to $-\frac{i}{\theta} \epsilon_{i j}\left[\hat{x}_{j}, \hat{f}\right]$. With the operator-valued fields, the action can be written as

$$
L=-\frac{2 \pi \theta}{g^{2}} \operatorname{tr}\left[\frac{1}{4} F_{\mu \nu} F^{\mu \nu}+D_{\mu} \phi\left(D^{\mu} \phi\right)^{\dagger}+\frac{\lambda}{2}\left(\phi \phi^{\dagger}-v^{2}\right)^{2}\right]
$$

where hats are dropped for simplicity and the derivative notation is understood as $\partial_{i} f \equiv-\frac{i}{\theta} \epsilon_{i j}\left[x_{j}, f\right]$. The system is invariant under the gauge transformation,

$$
A_{\mu}^{\prime}=U^{\dagger} A_{\mu} U+i U^{\dagger} \partial_{\mu} U, \quad \phi^{\prime}=U^{\dagger} \phi
$$

where the gauge group element $U$ satisfies

$$
U^{\dagger} U=U U^{\dagger}=I
$$

Using the translational invariance of the system, the Hamiltonian can be constructed as [2]

$$
H=\frac{2 \pi \theta}{g^{2}} \operatorname{tr}\left[\frac{1}{2}\left(E^{2}+B^{2}\right)+D_{t} \phi\left(D_{t} \phi\right)^{\dagger}+D_{i} \phi\left(D_{i} \phi\right)^{\dagger}+\frac{\lambda}{2}\left(\phi \phi^{\dagger}-v^{2}\right)^{2}\right],
$$

and the static equations of motion read

$$
\begin{gathered}
D_{i} D_{i} \phi-\lambda\left(\phi \phi^{\dagger}-v^{2}\right) \phi=0, \\
\epsilon_{i j} D_{j} B=J_{i} \equiv i\left[\phi\left(D_{i} \phi\right)^{\dagger}-D_{i} \phi \phi^{\dagger}\right],
\end{gathered}
$$

where we use the gauge $A_{0}=0$ and set $\partial_{t}=0$. Here the magnetic field transforms, under the gauge transformation, covariantly as $B^{\prime}=U^{\dagger} B U$ and the covariant derivative on an adjoint field $H$ is understood as $D_{\mu} H=\partial_{\mu} H-i\left[A_{\mu}, H\right]$. Let us introduce the creation and annihilation operators by $c^{\dagger} \equiv \frac{1}{\sqrt{2 \theta}}(x+i y)$ and by $c \equiv \frac{1}{\sqrt{2 \theta}}(x-i y)$, which satisfy $\left[c, c^{\dagger}\right]=1$. To represent arbitrary operators in the Hilbert space we shall use the occupation number basis by $G=\sum g_{m n}|m\rangle\langle n|$ with the number operator $\hat{n}=c^{\dagger} c$. We will further denote $A=A_{x}-i A_{y}, \partial_{-} G \equiv\left(\partial_{x}-i \partial_{y}\right) G=\frac{\sqrt{2}}{\sqrt{\theta}}[c, G]$ and $\partial_{+} G \equiv\left(\partial_{x}+i \partial_{y}\right) G=-\frac{\sqrt{2}}{\sqrt{\theta}}\left[c^{\dagger}, G\right]$.

We first present a unit flux static solution, where the flux is defined by $\Phi=\theta \operatorname{tr} B$. (This corresponds to $\Phi=\frac{1}{2 \pi} \int d^{2} x B$ when translated back to the language of ordinary functions.) The unit flux solution is

$$
\begin{aligned}
& \phi=v \sum_{n=0}^{\infty}|n+1\rangle\langle n|, \\
& A=\frac{\sqrt{2}}{i \sqrt{\theta}}\left(c-\frac{\sqrt{\hat{n}}}{\sqrt{\hat{n}+1}} c\right)=\frac{\sqrt{2}}{i \sqrt{\theta}} \sum_{n=0}^{\infty}(\sqrt{n+1}-\sqrt{n})|n\rangle\langle n+1| .
\end{aligned}
$$

It is simple to check that

$$
B=\frac{1}{\theta}|0\rangle\langle 0|,
$$

with which $\Phi=1$. Furthermore $D_{ \pm} \phi=0$ and $D_{ \pm} B=0$, so the second equation of motion in (11) is satisfied. Using $D^{2} \phi=D_{-} D_{+} \phi-B \phi$, one may show that $D^{2} \phi=0$ and $\left(\phi \phi^{\dagger}-v^{2}\right) \phi=0$, so the first equation in (11) is also fulfilled. 
The flux number of solution corresponds to $\Phi=1$. Thus the solution describes a static localized vortex. Since $|n\rangle\langle n|$ is invariant under rotation, our solution is axially symmetric. (Note here that $|n\rangle\langle n|$ is mapped to the function $2(-1)^{n} L_{n}\left(\frac{2 r^{2}}{\theta}\right) e^{-\frac{r^{2}}{\theta}}$ where $L_{n}(z)$ is the n-th order Laguerre polynomial.) By evaluating the Hamiltonian, one finds that the energy of the solution is

$$
E_{\text {one }}(v, \theta)=\frac{\pi}{g^{2}}\left(\frac{1}{\theta}+\lambda \theta v^{4}\right) \geq \frac{2 \pi}{g^{2}} \sqrt{\lambda} v^{2} .
$$

When $\lambda=1$, the theory allows so called Bogomol'nyi bound as discussed in Ref. [2]. In fact it is straightforward to verify that the energy functional can be expressed as a complete squared form plus a topological term by

$$
H=\frac{\pi \theta}{g^{2}} \operatorname{tr}\left[\left(B+\left(\phi \phi^{\dagger}-1\right)\right)^{2}+2\left(D_{+} \phi\right)\left(D_{+} \phi\right)^{\dagger}+\epsilon_{i j} D_{i} J_{j}+2 v^{2} B\right] \geq \frac{2 \pi v^{2}}{g^{2}} \Phi,
$$

where we omitted the kinetic terms involving $E_{i}$ and $D_{t} \phi$. The saturation of the bound occurs once the Bogomol'nyi equations,

$$
D_{+} \phi=0, \quad B=1-\phi \phi^{\dagger},
$$

are satisfied. When $\lambda=1$, the bound in (14) agrees with the Bogomol'nyi bound that is an absolute energy bound for one vortex solution. Hence when $v^{2}=\frac{1}{\theta}$ and $\lambda=1$, the solution should be a BPS solution. Indeed for the specific value of $v$, one can check that the solution satisfies the BPS equations. This BPS solution is clearly stable because they saturate the energy bound set by the topological quantity. For $\lambda \neq 1$ or $v^{2} \neq \frac{1}{\theta}$, it is not clear at this point whether these solutions are classically stable or not. For generic $\lambda$, the saturation of the bound in (14) can be achieved when $v^{2}=1 /(\sqrt{\lambda} \theta)$, whose physical implications are again not clear.

There are some physically equivalent configurations connected by gauge transformations. For example, $B=\frac{1}{\theta}|k\rangle\langle k|$ solution with $k>0$ can be obtained by the gauge transformation with

$$
U=1-|k\rangle\langle k|-| 0\rangle\langle 0|+| k\rangle\langle 0|+| 0\rangle\langle k| .
$$

Also rather simple extension of above solution comes from the translational invariance of the system. Our solution describes a vortex located at the origin and the explicit form of vortex at $\left(a_{x}, a_{y}\right)$, can be constructed with help of the translation generators $T_{i}=-\frac{1}{\theta} \epsilon_{i j}\left[x_{j},\right]$. For the above solution or solutions below, they will be just given as $A^{\prime}=e^{-i a_{i} T_{i}} A$ and $\phi^{\prime}=e^{-i a_{i} T_{i}} \phi$.

We now turn to the case of $\Phi=m(m \geq 1, m \in Z)$ vortex solutions. The solutions read

$$
\begin{aligned}
& \phi=v \sum_{n=0}^{\infty}|n+m\rangle\langle n|, \\
& A=\frac{\sqrt{2}}{i \sqrt{\theta}}\left(c-K_{m} \frac{\sqrt{\hat{n}-m+1}}{\sqrt{\hat{n}+1}} K_{m} c\right)=\frac{\sqrt{2}}{i \sqrt{\theta}} \sum_{n=0}^{\infty} \sqrt{n+1}(|n\rangle\langle n+1|-| n+m\rangle\langle n+m+1|),
\end{aligned}
$$

where the operator $K_{m}$ denotes the projection operator $1-\sum_{n=0}^{m-1}|n\rangle\langle n|$. Similar to the case of one vortex, the static equations of motion can be checked explicitly. Especially, they again satisfy $D_{ \pm} \phi=0$ and the magnetic field $B$ is given by

$$
B=\sum_{n=0}^{m-1}|n\rangle\langle n| .
$$


Thus the solution carries the vortex number $\Phi=m$. The energy for the solution is evaluated as

$$
E_{m}=m E_{\text {one }}
$$

The saturation of the Bogomol'nyi bound occurs at $v^{2}=\frac{1}{\theta}$ with $\lambda=1$ and, for these values of parameters, one may check that the solutions satisfy the BPS equations.

Interestingly, the above gauge field $A$ is also static solution of the pure gauge theory [⿶], where the static equation reads $D_{i} B=0$. We note that the magnetic field of the solution is well localized with a size of order $\sqrt{\theta}$. This kind of localized pure magnetic solutions cannot be static in the ordinary pure $\mathrm{U}(1)$ theory. This is because nothing prevents the magnetic field from spreading out, which will result in a configuration of the lower energy in the ordinary $\mathrm{U}(1)$ Maxwell theory.

A few comments are in order. First of all, these multi-vortex solutions are static. For the case when the Bogomol'nyi bound is saturated, the existence of static vortex solution has clear physical reasons. In the commutative case, there is of course no long range interactions because the Higgs and photons become massive by the Higgs mechanism. However, the vortices even may overlap completely and, hence, the cancellation of short-ranged interaction is necessary in order to have static configurations. When the BPS equations are satisfied, the forces between the vortices cancel out indeed. Namely, when we have vortices, there is in general current circulating around the vorticities. Because there is also local magnetic field, these combine to give local Lorenz force density. Also there is force contribution from Higgs gradient and the Higgs potential. These contributions should be canceled whenever we have a static configuration of vortices. The BPS equations may be regarded as such conditions that guarantee the cancellation of the local force density. In case of the static noncommutative multi-vortex, the current density $J_{i}$ is zero and, also, the contributions from the Higgs gradient and potential vanish since $\left(D_{i} \phi\right) \phi^{\dagger}=0$ and $\left(\phi \phi^{\dagger}-1\right) \phi=0$. Hence the existence of the static multi-vortex solutions indeed relies upon vanishing of local force densities. Secondly, one may alternatively consider the theory with $V=\frac{\lambda}{2} \operatorname{tr}\left(\phi^{\dagger} \phi-v^{2}\right)^{2}$, witch is still gauge invariant. It is then simple to show that the solution above solves again this new theory. However the energy will become different. This is due to the fact that $\operatorname{tr}\left(\phi^{\dagger} \phi-v^{2}\right)^{2} \neq \operatorname{tr}\left(\phi \phi^{\dagger}-v^{2}\right)^{2}$ in general. Thirdly we can check explicitly that the topological number $\Phi(=m)$ of the multi-vortex agrees with the index[2], 0, 8]

$$
I \equiv \operatorname{dim}\left[\operatorname{ker} \phi^{\dagger}\right]-\operatorname{dim}[\operatorname{ker} \phi]
$$

on our solutions.

Noncommutative False Vacuum Bubble with Magnetic Flux: Recently, the localized vacuum bubble solitons are found in noncommutative field theories 6]. They are localized false vacuum bubble of the relevant theory, where the noncommutativity of coordinates prevents its collapse to a zero size as dictated the uncertainty relation $\Delta x \Delta y \geq \theta$. Thus the bubble has a size of order $\theta$ and, outside of false vacuum region of core, the field configuration approaches to its stable minimum. Until now, no closed form solutions are found for finite $\theta[9]$.

We shall present similar false vacuum bubble solutions in the Abelian-Higgs model with $m$ magnetic flux. To have false vacuum bubbles, we consider the system in (7) but with more general potentials of the form $\frac{2 \pi \theta}{g^{2}} V(\xi)$ with $\xi \equiv \phi \phi^{\dagger}$ that is consistent with the gauge symmetry. We further assume that the true minima of potential are located at $\xi=0$ with $V(0)=0$ and there is 
at least one other local minimum. The static equations of motions are given by

$$
D_{i} D_{i} \phi-V^{\prime}\left(\phi \phi^{\dagger}\right) \phi=0
$$

with $\epsilon_{i j} D_{j} B=J_{i}$ that is the same as before. The static solutions with $m(\geq 1)$ magnetic flux are given by

$$
\begin{aligned}
\phi & =\phi_{0}|0\rangle\langle 0|, \\
A & =-i \frac{\sqrt{2}}{\sqrt{\theta}}\left(c-e^{i \nu} K_{m} \frac{\sqrt{\hat{n}-m+1}}{\sqrt{\hat{n}+1}} K_{m} c\right),
\end{aligned}
$$

where $\nu$ is real constant and $\phi_{0}$ is determined by an algebraic equation by

$$
1+\theta V^{\prime}\left(\xi_{0}\right)=0
$$

with $\xi_{0}=\left|\phi_{0}\right|^{2}$. To show that they are indeed satisfying the equations of motion, we note that $B=\sum_{n=0}^{m-1}|n\rangle\langle n|, D_{+} \phi=0$ and $D_{-} \phi=-\frac{\sqrt{2}}{\sqrt{\theta}} \phi_{0}|0\rangle\langle 1|$. Because $\left(D_{-} \phi\right) \phi^{\dagger}=0$, and $J_{i}=D_{i} B=0$. The equation of motion in (22) leads to the condition of (24). The flux number is $\Phi=m$ and the energy is given by

$$
E=\frac{2 \pi}{g^{2}}\left(\frac{m}{2 \theta}+\xi_{0}+\theta V\left(\xi_{0}\right)\right) .
$$

When $\theta$ is large enough, the values of $\xi_{0}$ satisfying the condition is given by the extremum of the potential, which is expected from the analysis of Ref. [6] for the scalar noncommutative solitons. Also the leading term of energy in the large $\theta$ limit is again consistent with the analysis given in Ref. [6].

Although one may consider more general potentials easily, we illustrate further properties of the solutions with

$$
V(\xi)=\beta\left[2 \xi^{3}-3(a+b) \xi^{2}+6 a b \xi\right]
$$

where we take $b>a>0$. Noting $V^{\prime}(\xi)=6 \beta(\xi-a)(\xi-b)$. The condition (24) becomes now $1+6 \theta \beta(\xi-a)(\xi-b)=0$. Hence when $\theta \rightarrow \infty$, the solutions are $\left|\phi_{0}\right|^{2}=a, b$. Here $a$ corresponds to the classically unstable soliton, while $b$ gives a classically stable one. For general $\theta$, the solutions read

$$
2 \xi_{0}^{ \pm}=a+b \pm \sqrt{(b-a)^{2}-\frac{2}{3 \theta \beta}}
$$

for $(b-a)^{2}-\frac{2}{3 \theta \beta} \geq 0$. Otherwise, there are no solutions satisfying the condition. The branch $\xi_{0}^{-}$is classically unstable because it maximizes the energy in (25).

Finally, one may find more general solutions as follows. Let us consider the Higgs and gauge fields of the form,

$$
\begin{aligned}
\phi & =\sum_{k=0}^{m-1} \phi_{k}|k\rangle\langle k| \\
A & =-i \frac{\sqrt{2}}{\sqrt{\theta}}\left(c-e^{i \nu} K_{m} \frac{\sqrt{\hat{n}-m+1}}{\sqrt{\hat{n}+1}} K_{m} c\right) .
\end{aligned}
$$


The static equations of motion (22) are then reduced to

$$
\begin{gathered}
J_{+}=\frac{\sqrt{2}}{i \sqrt{\theta}} \sum_{k=0}^{m-2}\left[\phi_{k+1} \phi_{k}^{*}|k+1\rangle\left\langle k\left|-\phi_{k} \phi_{k+1}^{*}\right| k\right\rangle\langle k+1|\right]=0, \\
{\left[2 k+1+\theta V^{\prime}\left(\xi_{k}\right)\right] \xi_{k}=0,}
\end{gathered}
$$

where $J_{+} \equiv J_{1}+i J_{2}$ and $\xi_{k}=\left|\phi_{k}\right|^{2}$. The first equation implies that $\phi_{k} \phi_{k+1}^{*}=0$ for all $k$. Namely, for any given nonvanishing $\phi_{k}$, the neighboring elements, $\phi_{k \pm 1}$ ought to be zero. The second equation is again purely algebraic and can be solved once the explicit form of the potential is given.

In this note, we construct exact multi-vortex solutions of the noncommutative Abelian Higgs theory. Further considering the Abelian-Higgs theory with more complicated scalar potential, we find static false vacuum bubble solutions with $m$ magnetic flux. The mechanism behind the existence of such a static multi-vortex solution is not fully identified. Hence the way we get the solutions was not quite systematic. In this respect, it is not clear whether we have obtained full category of such solutions. This requires further study.

Not to mention, the low energy moduli dynamics of our multi-solitons will be particularly of interest. Similar multi-vortex solutions are also expected in the noncommutative version of the nonrelativistic scalar theory [10] or the relativistic gauge theory [1]] coupled to Chern-Simons gauge theory and a work is in progress [12]. It is also quite obvious that, $D+1$ dimensional Abelian-Higgs type theory, trivial embedding of our solutions will give extended objects of dimension $D-2$. Our solutions would also be relevant to understanding the tachyon condensation in string theory [8, 13].

Note added: The detailed stability analysis of the pure flux solutions in the U(1) Maxwell theory has recently appeared in [14]. In this paper, it is shown that there are tachyonic modes in their fluctuation spectra.

Acknowledgment This work is supported in part by KOSEF 1998 Interdisciplinary Research Grant 98-07-02-07-01-5. We like to thank the enlightening discussions with K. Lee and J. H. Yee. We also would like to thank the hospitality of YVRC at Yonsei University where part of this work is done.

\section{References}

[1] H. B. Nielsen and P. Olesen, Nucl. Phys. B61 (1973) 45.

[2] D. P. Jatkar, G. Mandal and S. R. Wadia, hep-th/0007078.

[3] L. Alvarez-Gaume and S. R. Wadia, hep-th/0006219.

[4] A. P. Polychronakos, hep-th/0007043.

[5] D. J. Gross and N. A. Nekrasov, hep-th/0007204; B.-H. Lee, K. Lee and H.S. Yang, hep-th/0007140;

[6] R. Gopakumar, S. Minwalla and A. Strominger, JHEP 05 (2000) 020. 
[7] K. Furuuchi, hep-th/0005199.

[8] E. Witten, hep-th/0006071.

[9] C.-G. Zhou, hep-th/0007255.

[10] R. Jackiw and S.Y. Pi, Phys. Rev. Lett. 64 (1990) 2969.

[11] J. Hong, Y. Kim and P. Y. Pac, Phys. Rev. Lett 64 (1990) 2230; R. Jackiw and E. Weinberg, Phys. Rev. Lett 64 (1990) 2234.

[12] D. Bak, S. K. Kim, K.-S. Soh and J. H. Yee, in preparation.

[13] J. A. Harvey, P. Kraus, F. Larsen and E. J. Matinec, JHEP 07 (2000) 042; K. Dasgupta, S. Mukhi, and G. Rajesh, JHEP 06 (2000) 022.

[14] M. Aganagic, R. Gopakumar, S. Minwalla and A. Strominger, hep-th/0009142. 